\title{
Importância da tomografia de coerência óptica e do eletrorretinograma na retinosquise senil
}

\author{
Importance of optical coherence tomography and \\ electroretinogram in senile retinoschisis
}

Regina Halfeld Furtado de Mendonça', Otacílio Oliveira Maia Júnio², Fábio Gasparin³, Luciana Duarte Rodrigues ${ }^{4}$, Walter Yukihiko Takahash

\section{ResUmo}

O diagnóstico da retinosquise pode ser, muitas vezes, difícil por causa das formas clínicas atípicas. Freqüentemente, os exames complementares são necessários para a confirmação diagnóstica. $\mathrm{O}$ descolamento de retina deve sempre ser considerado no diagnóstico diferencial. Relato de caso: Paciente do sexo feminino, negra, com 61 anos de idade, assintomática. Na fundoscopia do olho direito, detectou-se uma lesão retiniana superior periférica que se estendia ao pólo posterior. O olho contralateral era normal. Na ultra-sonografia do olho direito, evidenciou-se uma membrana de alta reflexividade. No campo visual, detectou-se um defeito absoluto no campo inferior. O OCT, com scan centrado na ruptura da camada externa, demonstrou a separação da estrutura da retina. O ERG escotópico revelou uma nítida redução da amplitude da onda $\underline{b}$ no olho direito em relação ao olho contralateral (resposta com "onda negativa" no olho direito). O ERG multifocal era sem alterações nos dois olhos. Nos casos duvidosos, os exames complementares são indispensáveis. O OCT demonstrou morfologicamente a alteração. O ERG e a presença do escotoma absoluto no campo inferior confirmaram a alteração funcional da retina. A presença da "onda negativa" demonstra o comprometimento da função das camadas internas da retina através da redução da onda $\underline{b}$ e a função normal dos fotorreceptores com a presença da onda a inalterada. Casos duvidosos devem sempre ser investigados e estudados cuidadosamente para que tenhamos um diagnóstico de certeza e, conseqüentemente, uma conduta médica mais efetiva evitando procedimentos que possam eventualmente comprometer a visão.

Descritores: Retinosquise/diagnóstico; Eletrorretinografia/métodos; Descolamento retiniano; Tomografia de coerência óptica; Idoso

\footnotetext{
'Doutora, Assistente da Fundação Faculdade de Medicina da Universidade de São Paulo - USP - São Paulo (SP), Brasil; Pesquisadora da Faculdade de Medicina da Università "La Sapienza" - Roma - Italia;

2 Pós-graduando do Setor de Retina e Vítreo do Departamento de Oftalmologia do Hospital das Clínicas da Faculdade de Medicina da Universidade de São Paulo USP - São Paulo (SP), Brasil;

${ }^{3}$ Residente do Departamento de Oftalmologia do Hospital das Clínicas da Faculdade de Medicina da Universidade de São Paulo - USP - São Paulo (SP), Brasil;

${ }^{4}$ Pós-graduanda da Faculdade de Medicina da Universidade de São Paulo - USP - São Paulo (SP), Brasil;

${ }^{5}$ Doutor, Assistente e Chefe do Setor de Retina e Vítreo do Departamento de Oftalmologia do Hospital das Clínicas da Faculdade de Medicina da Universidade de São Paulo - USP - São Paulo (SP), Brasil.
}

Recebido para publicação em: 01/03/2007 - Aceito para publicação em 24/10/2007 


\section{INTRODUÇÃO}

A retinosquise é um processo degenerativo da retina com separação de suas lamelas e formação de cistos entre suas camadas. O diagnóstico da retinosquise pode ser, muitas vezes, difícil por causa das formas clínicas atípicas ${ }^{(1)}$. A retinosquise senil, também chamada adquirida, é bilateral em 50 a $80 \%$ dos pacientes afetados ${ }^{(2)}$, acometendo o quadrante inferotemporal $^{(3-4)}$ e está comumente associada com hipermetropia ${ }^{(2)}$. É geralmente assintomática e não pro$\operatorname{gressiva}^{(4)}$. Na retinosquise degenerativa típica, a retina se divide na camada plexiforme externa ${ }^{(2)}$. $\mathrm{Na}$ oftalmoscopia com depressão escleral, a camada externa aparece irregular, manchada e a camada interna é fina, elevando-se de forma ovalada e lisa ${ }^{(2)}$. Muitas vezes, os exames complementares são necessários para a confirmação diagnóstica ${ }^{(3,5)}$. O descolamento de retina deve sempre ser considerado no diagnóstico diferencial ${ }^{(2,6,7)}$. Alguns autores utilizaram a fotocoagulação com laser de argônio nos casos duvidosos, e a reação decorrente dessa fotocoagulação pode diferenciar a retinosquise do descolamento de retina ${ }^{(7)}$. A ressecção das camadas internas demonstraram aumentar o risco de formação de membranas epirretinianas ${ }^{(8)}$. O tratamento deve ser considerado apenas em último caso, quando o paciente apresenta sintomas decorrentes do descolamento de retina progressivo $^{(4)}$. No presente trabalho, relata-se um caso difícil onde a utilização da OCT e eletrofisiologia contribui para o estabelecimento diagnóstico.

\section{Relato do Caso}

Paciente do sexo feminino, negra, com 61 anos de idade compareceu ao Ambulatório de Oftalmologia da Prefeitura Municipal de São José dos Campos para exame de rotina. Ao exame, detectou-se acuidade visual de Snellen de 20/20 em ambos os olhos e J1 (tabela de Jaeger) com $+2,50$ para perto. A paciente tinha J1 de visão para perto. Foram normais os exames da motilidade ocular, biomicroscopia do segmento anterior e tonometria de aplanação. Na fundoscopia do olho direito, detectou-se uma lesão retiniana superior periférica que se estendia ao pólo posterior. O olho contralateral era normal. A paciente foi encaminhada imediatamente ao Ambulatório de Oftalmologia do Hospital das Clínicas da Faculdade de Medicina da Universidade de São Paulo para investigação diagnóstica, onde foram realizados os exames de retinografia, [evidenciando no olho direito, retinosquise que se estende da periferia até o pólo posterior, lesões esbranquiçadas na camada interna (reação glial) e ruptura da camada externa da retinosquise] (Figura 1); ultra-sonografia (evidenciando no Modo A, eco de alta refletividade e prega fixa com limite anterior pouco convexo e no Modo B, imagem cística, anecóica e imóvel) (Figura 2); campo visual (mostrando uma contração periférica parcial inferior das isópteras formadas com as miras V4e , I4e , I2e; uma constrição da isóptera I1e e um discreto aumento da mancha cega , que foi traçada com a mira I4e. Como na área do defeito não houve respostas aos estímulos com mira V4e, pode-se afirmar que se trata de um defeito absoluto no campo inferior, correspondente à área com retinosquise observada no fundo de olho) (Figura 3); tomografia de coerência óptica (demonstrando a separação da estrutura retínica) (Figura 4) e exames eletrodiagnósticos (ERG de campo total demonstrando uma nítida redução da amplitude da onda b no olho direito em relação ao olho contralateral) (Figura 5).

\section{Discussão}

Alguns casos de Retinosquise são de difícil diagnóstico. Exames complementares são necessários para a confirmação e exclusão de outras doenças que se assemelham com o quadro clínico. No presente caso, ressalta-se a importância dos exames complementares principalmente no diagnóstico diferencial com descolamento de retina.

Ao exame de fundo de olho e na retinografia, foram evidenciadas lesões retinianas compatíveis com retinosquise, sugerindo uma ruptura na camada externa. É importante ressaltar que normalmente as alterações são bilaterais, acometem geralmente a região inferior da retina ${ }^{(3)}$ e raramente se estendem ao pólo posterior. No presente caso, a alteração era unilateral e comprometia a região superior da retina estendendo-se ao pólo posterior. Evidencia-se a importância da diferenciação diagnóstica com casos de descolamento de retina. Geralmente, na retinosquise, não há hemorragias ou "tabaco dust" comumente detectadas em casos de descolamento $^{(2)}$. Além disso, ressalta-se o aspecto morfológico da lesão sendo que, na retinosquise, encontra-se uma superfície lisa e, no descolamento, evidenciase uma superfície irregular e enrrugada ${ }^{(2)}$.

Na ultra-sonografia do olho direito, evidenciouse uma membrana de alta reflexividade de difícil diferenciação com o descolamento seroso de retina.

A presença do escotoma absoluto no campo inferior (Figura 3), correspondente à área com retinosquise 


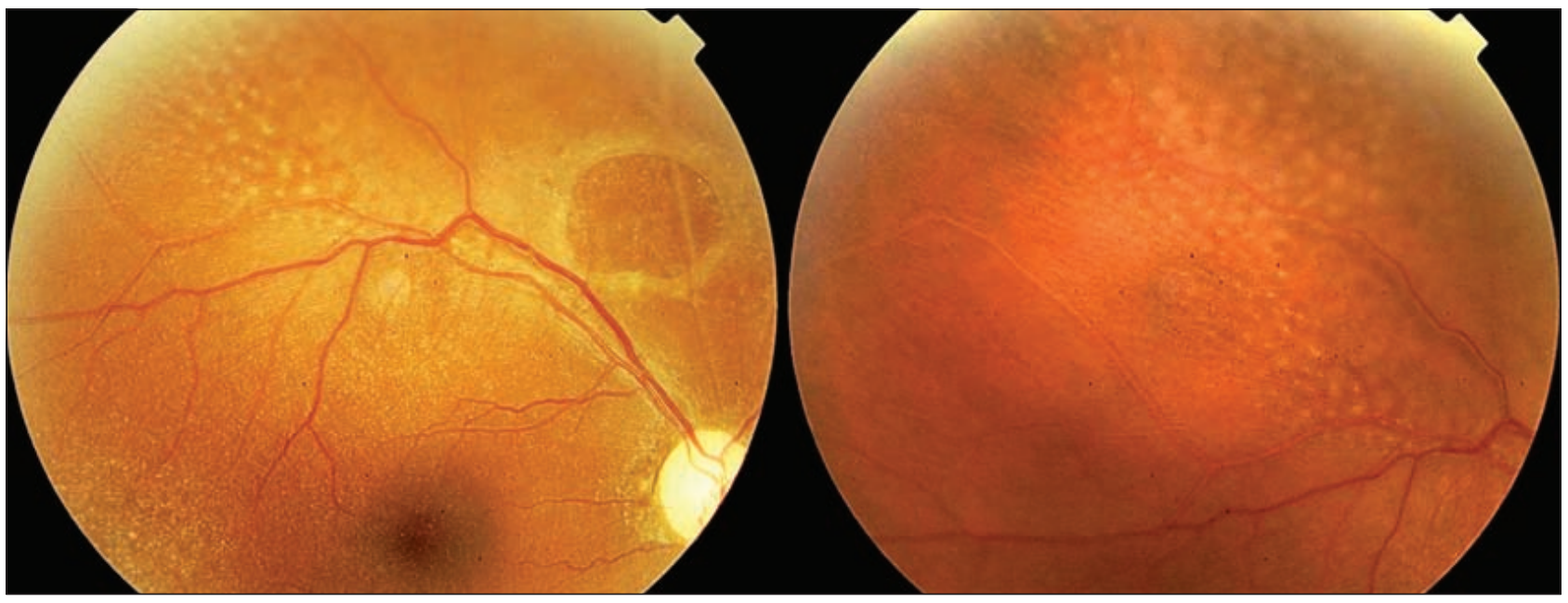

Figura 1: Retinografia do olho direito evidenciando retinosquise que se estende da periferia até o pólo posterior, lesões esbranquiçadas na camada interna (reação glial) e ruptura da camada externa da retinosquise

observada no fundo de olho afasta a possibilidade do descolamento de retina onde geralmente é detectado um escotoma relativo.

No OCT com o scan centrado na ruptura da camada externa (Figura 4), evidencia-se a separação das camadas da retina e inexistência de infiltração. Vários autores já demonstraram a importância do OCT na confirmação diagnóstica da retinosquise ${ }^{(3)}$. A importância do OCT, no diagnóstico diferencial entre retinosquise e descolamento de retina, também já foi relatada na literatura $^{(6)}$.

O ERG é um exame importante porque correlaciona-se bem com a intensidade do comprometimento retiniano e indica qual extrato retiniano está afetado ou entre quais camadas a "esquise" ocorre ${ }^{(9)}$. No presente caso (Figura 5), a presença da "onda negativa" ao estímulo máximo em condições escotópicas demonstra o comprometimento da função das camadas internas da retina através da redução da onda $\underline{b}$ e função normal dos fotorreceptores com a presença da onda a inalterada. Acredita-se que, no caso descrito, tenha ocorrido um comprometimento inicial das células de Müller demonstrado pela redução da onda $\underline{b}^{(10)}$. Essa redução normalmente grande da onda $\underline{b}^{(1,1)}$ com onda a normal ${ }^{(1)}$ é uma alteração típica da retinosquise juvenil, embora se saiba atualmente que a "onda negativa" do eletrorretinograma difuso não seja um sinal patognomônico de retinosquise ${ }^{(12)}$. De qualquer modo,não é o resultado esperado em casos de descolamento de retina onde as ondas a e b bão igualmente comprometidas ${ }^{(13)}$, demonstrando um mal funcionamento dos fotorreceptores e das camadas internas da retina. A normalidade do eletrorretinograma multifocal demonstra a integridade da região foveal. A retinosquise

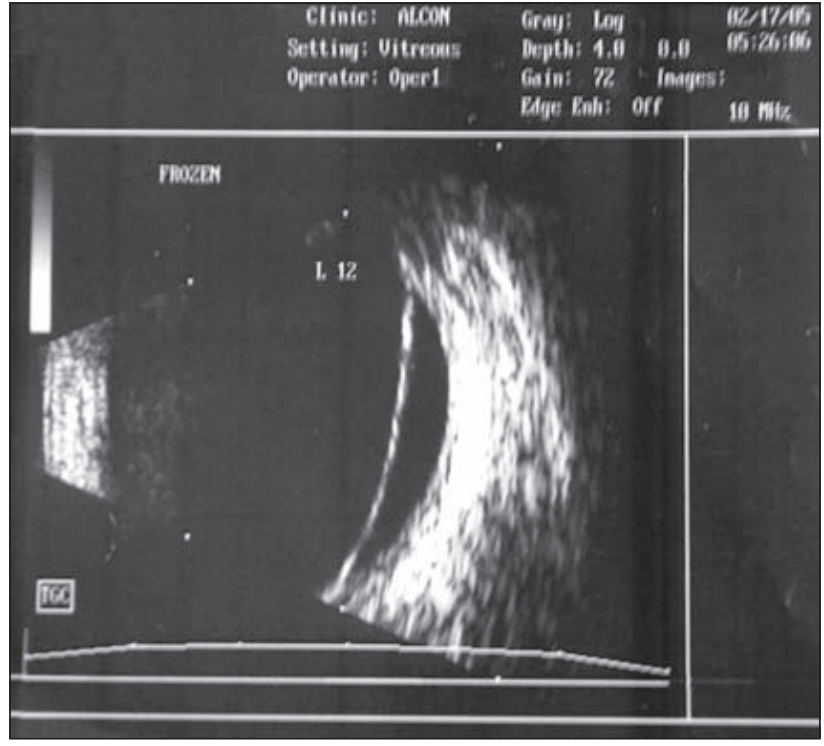

Figura 2: Ultra-sonografia do olho direito (modo B), evidenciando uma membrana de alta reflexividade de difícil diferenciação entre descolamento de retina seroso e retinosquise

periférica não reduz o ERG focal nos estágios onde não existem alterações visíveis de atrofia do epitélio pigmentar da retina ${ }^{(14)}$

Ressalta-se a importância do OCT e do eletrorretinograma na confirmação diagnóstica de certas formas atípicas de retinosquise. No presente caso, esses dois métodos associados ao campo visual foram responsáveis pela confirmação diagnóstica. Alguns autores consideram o tratamento com laser de argônio eficaz na prevenção das complicações ${ }^{(15)}$. Por outro lado, muitos autores compartilham da opinião de que é melhor adiar o tratamento até que as complicações apareçam ${ }^{(16)}$, sen- 


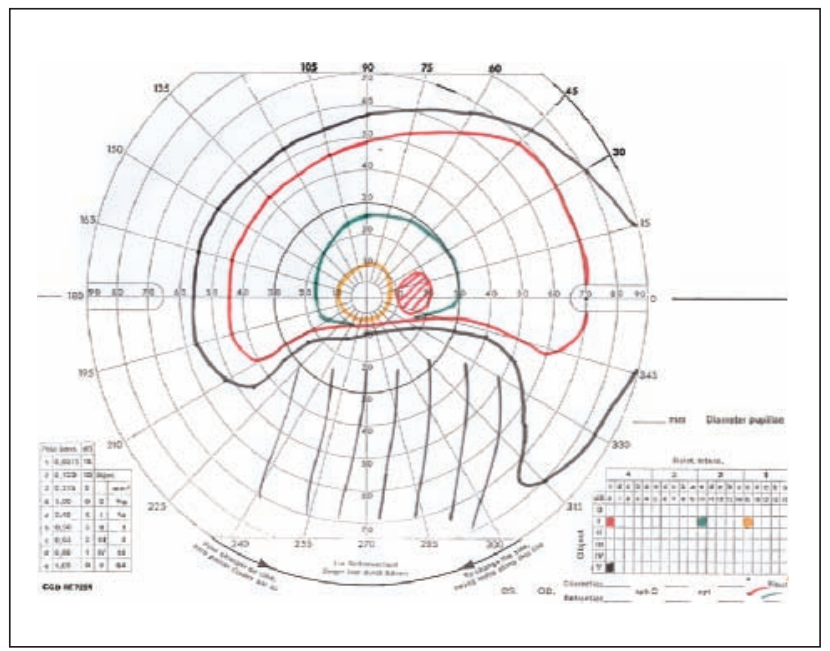

Figura 3: Campo visual manual realizado com perímetro de Goldmann (Haag-Streit). Foram traçadas isópteras com as miras V4e , I4e , I2e, Ile, com correção de $+3,0$ dioptrias nos $30^{\circ}$ centrais

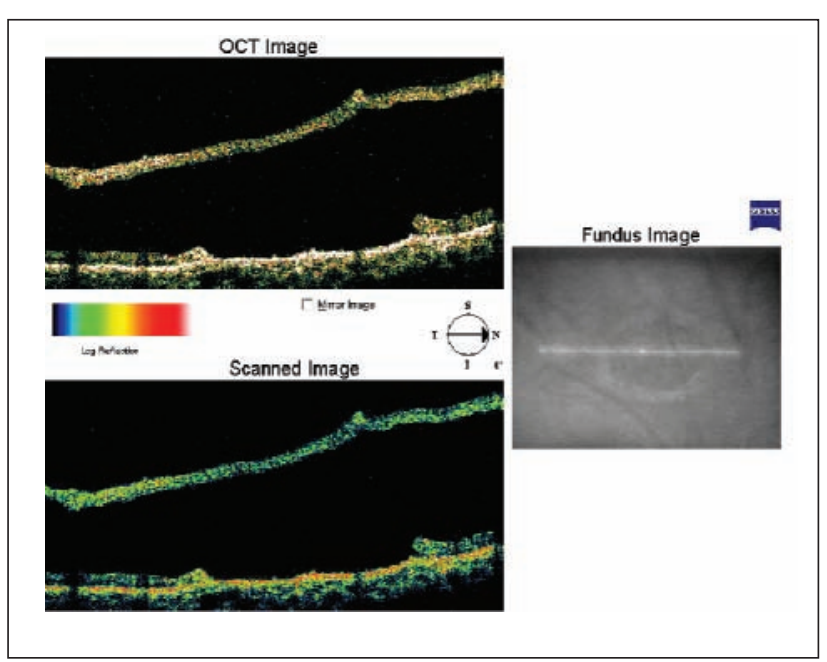

Figura 4: Tomografia de coerência óptica (Zeiss, Stratus OCT 3): scan centrado na ruptura da camada externa, demonstrando a separação da estrutura retínica

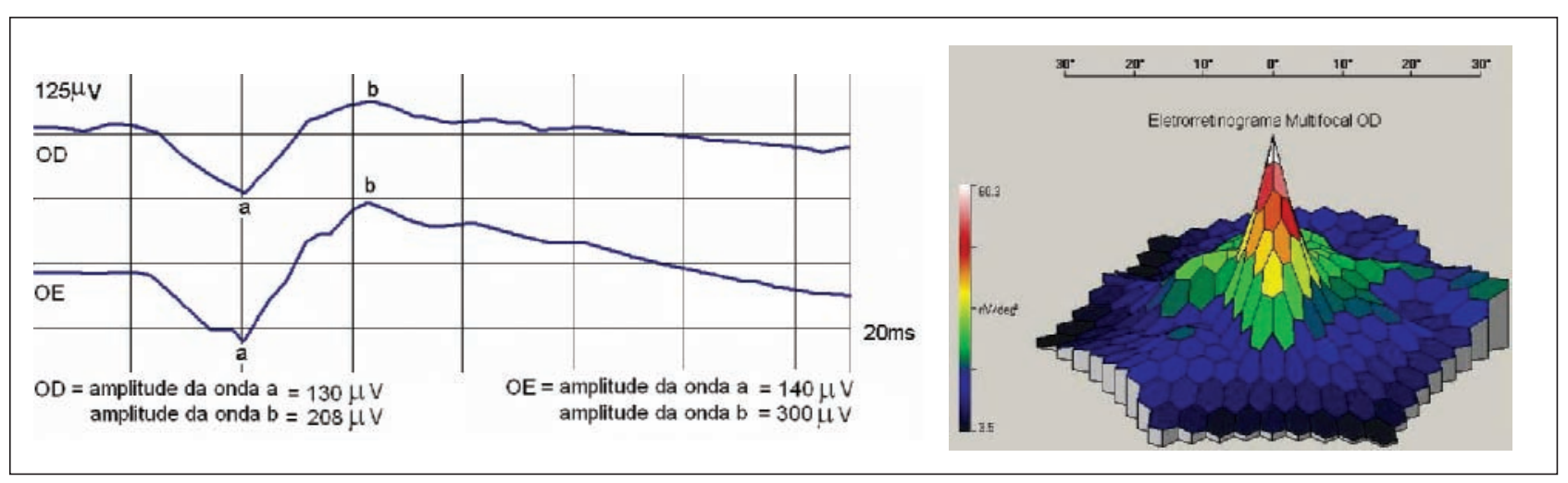

Figura 5: Eletrorretinograma escotópico (Retiscan - Roland Instruments - Electrophysiological diagnostic systems) demonstrando uma nítida redução da amplitude da onda b no olho direito em relação ao olho contralateral. Nota-se assim uma resposta com "onda negativa" no olho direito. O eletrorretinograma multifocal apresenta-se sem alteração nos dois olhos

do que o tratamento cirúrgico deverá ser feito somente e imediatamente nos casos progressivos e sintomáticos decorrentes de descolamento ${ }^{(16)}$ que ameaçam a mácu$\mathrm{la}^{(17)}$. É importante ressaltar que o risco provável do descolamento de retina, decorrente da retinosquise progredir ou tornar-se sintomático, é de apenas $0,07 \%{ }^{(18)}$. Casos duvidosos devem sempre ser investigados e estudados cuidadosamente para que tenhamos um diagnóstico de certeza e, conseqüentemente, uma conduta médica mais efetiva, evitando procedimentos que possam eventualmente comprometer a visão. Nos casos atípicos, o OCT e o ERG são importantes na confirmação diagnóstica, mudando eventual proposta terapêutica desnecessária.

\section{Abstract}

Retinoschisis diagnosis can be very difficult in atypical forms. Special exams are necessary to differentiate retinoschisis from other pathologies such as retinal detachment. Case Report: A 61-year-old black woman presented for routine examination. Fundus of the right eye reveled a superior periphery lesion extending to the posterior pole. The ultrassonography of the same eye showed a high reflective membrane. An inferior field absolute scotoma was detected. The OCT scan that was positioned at lesion in the outer retinal break demonstrated the fragmentation of the retinal layer. The scotopic ERG showed a reduction of the $\underline{b}$ wave amplitude of the right 
eye compared to the left eye (Negative electroretinogram). The multifocal ERG was normal in both eyes. The OCT demonstrated to be an useful toll to detect morphological alterations. The ERG and the absolute scotoma in the inferior field, demonstrated the retinal functional defect. A negative electroretinogram, showed a normal a wave and a reduced $\underline{b}$ wave demonstrating that the photoreceptor inner segments were relatively intact and that the initial damage in this disorder would seem to occur in the inner retinal layers. In doubtful cases especial exams are necessary and a careful investigation must confirm the diagnosis. The indications for treatment should be seriously analysed to avoid any intervention that can be harmful for the patient's prognosis.

Keywords: Retinoschisis/diagnosis; Electroretinography/methods; Retinal detachment, Tomography, optical coherence; Aged

\section{ReferÊNCIAS}

1. Yamaguchi K, Hara S. Autosomal juvenile retinoschisis without foveal retinoschisis. Br J Ophthalmol. 1989; 73(6): 470-3.

2. Moreira Jr CA, Ávila M. Retinopatia diabética. In: Moreira Jr CA, Ávila M. Manual CBO: Retina e vítreo. São Paulo: CBO; 2000. p.35-60.

3. Kamppeter BA, Jonas JB. Optical coherence tomography of a peripheral retinal schisis with an outer retinal layer break. Acta Ophthalmol Scand. 2004; 82(5): 574-5.

4. Byer NE. Long-term natural history study of senile retinoschisis with implications for management. Ophthalmology. 1986; 93(9): 1127-37.

5. Meirelles RL, Aggio FB, Costa RA, Farah ME. STRATUS optical coherence tomography in unilateral colobomatous excavation of the optic disc and secondary retinoschisis. Graefes Arch Clin Exp Ophthalmol. 2005; 243(1): 76-81.

6. Ip M, Garza-Karren C, Duker JS, Reichel E, Swartz JC, Amirikia A, Puliafito CA. Differentiation of degenerative retinoschisis from retinal detachment using optical coherence tomography. Ophthalmology. 1999; 106(3): 600-5. Comment in: Ophthalmology. 2000; 107(7):1211.
7. Landers MB 3rd, Robinson CH. Photocoagulation in the diagnosis of senile retinoschisis. Am J Ophthalmol. 1977; 84(1): 18-23.

8. Hoerauf H, Joachimmeyer E, Laqua H. Senile schisis detachment with posterior outer layer breaks. Retina. 2001; 21(6): 602-12.

9. Carr RE, Siegel IM. The vitreo-tapeto-retinal degenerations. Arch Ophthalmol. 1970; 84(4): 436-41 passim.

10. Peachey NS, Fishman GA, Derlacki DJ, Brigell MG. Psychophysical and electroretinographic findings in X-linked juvenile retinoschisis. Arch Ophthalmol. 1987; 105(4): 513-6.

11. Tanino T, Katsumi O, Hirose T. Electrophysiological similarities between two eyes with $\mathrm{X}$-linked recessive retinoschisis. Doc Ophthalmol. 1985; 60(2): 149-61.

12. Bradshaw K, Allen L, Trump D, Hardcastle A, George N, Moore A. A comparison of ERG abnormalities in XLRS and XLCSNB. Doc Ophthalmol. 2004; 108(2): 135-45.

13. Fishman GA. The electroretinogram in retinal disease. In: Fishman GA, Sokol S. Electrophysiologic testing in disorders of the retina, optic nerve, and visual pathway. San Francisco, CA: American Academy of Ophthalmology; c1990. p.1-89.

14. Piao CH, Kondo M, Nakamura M, Terasaki H, Miyake Y. Multifocal electroretinograms in X-linked retinoschisis. Invest Ophthalmol Vis Sci. 2003; 44(11): 4920-30.

15. Yassur Y, Feldberg R, Axer-Siegel R, Silverston B, Manor R, Ben-Sira I. Argon laser treatment of senile retinoschisis. Br J Ophthalmol. 1983; 67(6): 381-4.

16. Byer NE. Perspectives on the management of the complications of senile retinoschisis. Eye. 2002; 16(4): 359-64.

17. Clemens S, Busse H, Gerding H, Hoffmann P. [Treatment guidelines in various stages of senile retinoschisis]. Klin Monatsbl Augenheilkd. 1995; 206(2): 83-91 German.

18. Byer NE. The natural history of senile retinoschisis. Trans Sect Ophthalmol Am Acad Ophthalmol Otolaryngol. 1976; 81(3 Pt 1): 458-71.

ENDEREÇOS PARA CORRESPONDÊNCIA:

Regina Halfeld Furtado de Mendonça

Via Muzio Attendolo, 31

00176 - Roma - Itália

Tel/fax: 00 XX 390621701794

00 XX 393393735455

e-mail: halfeld@libero.it 